\title{
La préposition spatiale dynamique à travers (de) et les processus non-téliques Analyse sémantique de l'usage attesté au $\mathrm{XVI}^{\mathrm{e}}$ siècle $^{\mathrm{I}}$
}

\author{
Thomas Hoelbeek*
}

\section{INTRODUCTION}

La préposition analytique à travers appartient au groupe restreint des principaux marqueurs spatiaux qui caractérisent les relations dynamiques tout en étant dotés d'une polarité médiane. Elle est directionnelle et, comme par et via, décrit souvent une situation de parcours (Borillo 1998 : 85). Cependant, elle est plutôt non-relationnelle, parce qu'elle ne se combine que difficilement avec une référence à une voie de communication (Stosic 2005 : 201).

Dans cette contribution - qui prend pour cadre un projet de recherche plus ample concernant la structure sémantique et le fonctionnement actuel et ancien de toutes les prépositions contenant les mots français et italien travers ou traverso (voir Hoelbeek 2014) - nous proposons une analyse sémantique de l'usage qui était fait, au $\mathrm{XVI}^{\mathrm{e}}$ siècle, de la locution française à travers (de). Ce travail ne constitue donc qu'une première étape d'une étude qui examine des données provenant d'une période de quatre siècles situés entre la Renaissance et l'époque contemporaine (à savoir du $\mathrm{XVI}^{\mathrm{e}}$ au XIX $\mathrm{X}^{\mathrm{e}}$ siècle). Nous nous inspirons, entre autres, des travaux de Stosic (2002a; 2002b; 2005; 2007; 2009; 2012) qui a décrit de façon approfondie l'emploi spatial de à travers en français moderne, en le contrastant avec celui de par. La notion de " guidage » qu'il introduit, et qui serait centrale dans les emplois spatiaux de à travers, a l'avantage d'être moins

* Vrije Universiteit Brussel - Université Libre de Bruxelles. Courriel : Thomas.Hoelbeek@gmail.com

1 Nous tenons à remercier vivement Marc Dominicy pour ses suggestions précieuses et constructives. L’auteur assume pleinement la responsabilité des erreurs et omissions qui subsisteraient. 
ambiguë que la notion d' « obstacle ». Enfin, en nous fondant sur la typologie de Vet (1994), nous analyserons quelques occurrences dans lesquelles la nature télique ou non-télique du procès en cause peut prêter à discussion.

Tout en nous situant dans une perspective à la fois diachronique et cognitive, nous adoptons une approche fonctionnelle dans la tradition des travaux de, entre autres, Vandeloise (1986).

\section{REMARQUES METHODOLOGIQUES GENERALES}

Dans le cadre théorique de la linguistique cognitive, deux entités sont à distinguer dans la description de l'espace : l'entité à localiser (le sujet de la relation spatiale) et l'entité de référence (l'objet de la relation spatiale). Dans sa terminologie, que nous adoptons ici, Vandeloise (1986: 34) nomme l'entité à localiser la « cible » et l'entité de référence le « site ».

Le tableau (1) illustre le vocabulaire supplémentaire auquel nous recourons afin de distinguer et de classer les différents usages observés.

\begin{tabular}{|c|c|c|}
\hline \multicolumn{2}{|c|}{ Mouvement } & \multirow[b]{2}{*}{ Il y a un arbre en travers de la route } \\
\hline $\begin{array}{c}\text { Concret } \\
\text { Jean marche à travers la ville }\end{array}$ & $\begin{array}{c}\text { Fictif } \\
\text { Jean regarde à travers la fenêtre }\end{array}$ & \\
\hline \multicolumn{2}{|c|}{$\begin{array}{c}\text { Métaphoriquement projeté } \\
\text { Jean est passé à travers beaucoup de difficultés } \\
\text { Jean entend à travers les siècles les cris des gladiateurs }\end{array}$} & $\begin{array}{l}\text { Métaphoriquement projetée } \\
\text { Il s’est mis en travers de nos plans }\end{array}$ \\
\hline
\end{tabular}

$$
\text { Tableau (1) : Mouvement / position de la cible }
$$

Comme il ressort du tableau (1), les locutions contenant travers n'expriment pas exclusivement des situations dynamiques. De fait, à part des mouvements, on rencontre également des configurations statiques, ou positions. Concernant les configurations dynamiques, le tableau (1) montre que nous distinguons deux types de mouvement :

- un «mouvement concret» (terme emprunté à Stosic 2002b: 93) implique une transition réelle d'un endroit à un autre ${ }^{3}$;

- $\quad$ un " mouvement fictif » s'applique à une scène où l'appareil perceptuel de l'être humain ne peut pas détecter de mouvement. Le mouvement est donc seulement suggéré par le langage ; la cible est une entité fictive,

2 Dans le passée, à travers (de) était capable de décrire des situations dynamiques ainsi que statiques. Cependant, afin de faciliter la compréhension du lecteur moderne, les phrases que nous avons formulées pour illustrer les usages statiques contiennent l'expression en travers (de), aujourd'hui typiquement utilisée dans ce genre de situation.

3 Une position est toujours concrète, et se caractérise par une configuration spatiale où une entité occupe une certaine position par rapport à une entité de référence. 
ou non-tangible dans ces cas. Il peut s'agir d'un mouvement perceptuel (c'est-à-dire appartenant aux domaines de la vue, de l'ouïe ou du toucher) ou d'une autre sorte de mouvement (voir Talmy 2000: 99-175).

Enfin, le tableau (1) illustre la « projection » métaphorique (mapping en anglais) d'un mouvement (concret ou fictif) ou d'une position depuis un domaine-source tridimensionnel vers un domaine abstrait (par exemple le domaine temporel) où la cible et le site ne peuvent entretenir une relation spatiale dans un univers à trois dimensions.

\section{LES CONCEPTS FONCTIONNELS}

Dans l'approche dite fonctionnelle (cf. par exemple Vandeloise (1986) et Herskovits (1986)), l'espace est décrit au moyen de concepts fonctionnels inspirés par la forme du corps humain, la physique naïve, l'accès ou non à la perception, la rencontre potentielle et l'orientation générale et latérale. Ces concepts sont « liés à la connaissance extra-linguistique [de l'espace] que partagent les locuteurs d'une même langue » (Vandeloise 1986: 22).

Le concept d'orientation latérale s'avère particulièrement utile pour nos analyses. Ses principaux traits sont « la direction latérale » et « la perpendiculaire à l'orientation générale ». Cette dernière a comme traits « la direction frontale, la ligne du regard et la direction du mouvement » (Vandeloise 1986: 29).

Afin de capturer la sémantique de à travers en français moderne, Stosic (2002b: 106) a introduit la notion de "guidage » comme une alternative à la notion d' " obstacle ». Cette notion fonctionnelle correspond notamment au contrôle latéral qui est exercé sur la cible par le site.

Stosic se fonde sur la «dynamique des forces » (force dynamics) de Talmy (2000) afin de définir la cible comme un Agoniste qui exerce une force positive par rapport au site, qui joue le rôle d'un Antagoniste et s'oppose par l'effet d'une force passive de résistance. Il affirme qu'en français moderne, la préposition à travers est utilisée lorsque cette tension entre la force et la contre-force a lieu sur l'axe latéral. Cela signifie que l'opposition frontale n'est pas un facteur déterminant et que ce qui est nécessaire, c'est la présence et la saillance des deux pôles définis par rapport à l'orientation latérale (Stosic 2002b: 104-106).

\section{DESCRIPTION DU RESEAU SEMANTIQUE DE A TRAVERS (DE) AU XVI ${ }^{\mathrm{E}}$ SIECLE}

À ce stade, nous nous inspirons principalement de la typologie établie par Stosic (2002b), qui a proposé un inventaire systématique des usages synchroniques de à travers en français moderne. Sa notion de " guidage ", même si elle a été proposée afin de décrire les usages en français moderne de à travers, se révèle apte à décrire plusieurs usages de certaines prépositions au $\mathrm{XVI}^{\mathrm{e}}$ siècle. Cependant, toutes les configurations ne se laissent pas caractériser par cette notion. 
Nous allons donner ici un aperçu très condensé des emplois à distinguer, illustrés par des exemples provenant de FRANTEXT ${ }^{4}$.

\subsection{Emplois concrets (mouvements et positions) ${ }^{5}$}

Pour une grande partie des configurations que nous avons distinguées concernant les usages de à travers (de), la notion de " guidage " se révèle apte à caractériser les emplois observés ${ }^{6}$ :

(1) [...] mais c'est que quand ledit fumier estoit au champ par pilots, les pluyes qui sont survenues, ont passé à travers desdits pilots de fumier, [...]

(Bernard Palissy, Recepte véritable, 1563)

Par contre, pour d'autres types de sites, il faut recourir à d'autres notions. Il s'agit d'usages qui sont proches d'un des emplois actuels d'en travers (de), caractérisé, selon Somers (1988: 55), par un « concept de résistance [vue] comme une opposition à l'utilisation habituelle du site ». Souvent, la cible se trouve dans une position orthogonale par rapport au site. Quant à l'usage de à travers (de) au $\mathrm{XVI}^{\mathrm{e}}$ siècle, il n'est pas forcément caractérisé par une résistance, mais il y a bien un « rapport d'orthogonalité » :

(2) En la mer à travers ce Royaume ne sont que les isles des Exoires, qui sont bien troys cens lieues en la mer.

(Alphonse Jean, dit Fonteneau, Voyages avantureux du Capitaine Jan Alfonce, Sainctongeois, 1544)

Une dernière configuration présente un sens qui, aujourd'hui, semble être plutôt véhiculé par parmi. Il s'agit de situations où l'on décrit la position d'une cible (souvent au pluriel) sans préciser exactement cette position. Stosic décrit cet usage comme le «balayage » d'un site (Stosic 2002b: 94) ; la cible couvre toute la surface du site, mais ses éléments occupent, à l'intérieur du site, des positions différentes :

(3) Car il n’y a point de meilleur expédient en toutes les esmotions et soubslevemens des subjects contre leur Prince, que d'abbreger, et donner ordre de les esteindre de bonne heure, sans leur laisser tant soit peu de loisir de prendre pied pour se multiplier et accroistre : Autrement cela va tost en infiny, ny plus

\footnotetext{
4 La présente contribution est basée sur l'analyse d'un corpus d'occurrences provenant de FRANTEXT. En sélectionnant tous les ouvrages datant du XVI ${ }^{\mathrm{e}}$ siècle, nous avons obtenu un total de 157 occurrences (26 pour la période $1500-1549$ et 131 pour la période $1550-1599$ ). Faute de place nous ne pouvons pas entrer ici dans les détails des traits analysés pour chaque occurrence.

5 Dans le présent travail nous traitons toujours ensemble les mouvements et les positions.

6 Pour faciliter la lecture des exemples anciens, nous avons appliqué l'accentuation actuelle chaque fois que le mot concerné se trouvait noté, pour le reste, selon les conventions aujourd'hui en cours.
} 
ne moins qu’un feu bien allumé à travers un gros taz de fagots, ou autre menu bois.

(Blaise de Vigenère, L'Histoire de la décadence de l'Empire grec, et establissement de celuy des Turcs, comprise en dix livres par Nicolas Chalcondyle,

\subsection{Mouvement fictif}

Les usages perceptuels constituent la majorité des configurations qui expriment un mouvement fictif. Elles semblent toutes entretenir un lien intime avec le concept de « guidage ». Cependant, Stosic (Stosic 2002b: 240-247) remarque qu'on ne peut pas toujours facilement distinguer entre la cible et le site, dans la mesure où la cible n'est pas toujours encodée. Caractéristique des usages perceptuels est le fait que des situations linguistiquement présentées comme dynamiques se révèlent, dans la réalité, plutôt statiques : il y a donc un "mouvement fictif » (Talmy 2000). Il s'agit de situations où le «parcours perceptif » (Stosic 2002b: 245) est représenté par une ligne (la ligne du regard, par exemple) ; dès lors, ce mouvement fictif est forcément rectiligne. On pourrait établir ici un rapprochement avec le verbe traverser, qui « décrit le passage d'un côté à l'autre d'une entité » (Sarda 1999: 146). Cette " contrainte du parcours minimal » entraîne « la nécessité pour la cible qui se déplace d’atteindre le côté opposé du site » (Stosic 2002b: 124) :

(4) Sus, sus, mes compagnons, pour ceste heureuse paix, Faisons ouïr nos voix à travers l'air espais :

(Louis des Masures, David fugitif, 1566)

\subsection{Projection métaphorique}

De même, pour les emplois métaphoriques de à travers (de) la plupart des configurations se laissent décrire par la notion de « guidage » :

(5) Le roy d'Espagne void tout à travers et tousjours du Conseil de vostre maistre.

(René de Lucinge, Dialogue du François et du Savoysien, 1593)

On rencontre aussi des emplois du type «balayage » :

(6) Quant aux autres jeusnes qui se font à travers l'an, nous remonstrons que les povres ignorans, pensans faire service à Dieu, sacrifient à leurs idoles.

(Jean Calvin, Des scandales, 1550)

\section{LA TYPOLOGIE DE VET (1994)}


Vet (1994), se basant sur les travaux fondateurs de Vendler (1957; 1967), analyse différents types de configurations qui expriment des relations temporelles, et qui sont le résultat de l'interaction entre l'Aktionsart (mode d'action), l'aspect et les compléments de durée. Dans sa description, la structure sous-jacente à la phrase est définie comme une composition de plusieurs strates :

- la prédication-1a se compose du prédicat et du nombre approprié d'arguments ;

- la prédication-1b comporte la prédication-1a éventuellement enrichie d’un complément de durée ;

- la prédication-2 ajoute l'opérateur aspectuel à la prédication 1a ou 1b, avec la valeur « imperfectif », " perfectif » ou " parfait » (valeur non traitée par Vet) ;

- la prédication-3a est formée par la prédication-2 précédée de l’opérateur temporel ;

- la prédication-3b est formée par la prédication-3a augmentée d’un adverbe de temps.

Remarquons encore que l'aspect perfectif est défini comme indiquant que « l'assertion concerne un procès complet, le début et la fin compris », et que « le temps référentiel indique [...] le laps de temps auquel se rapporte l'assertion » (Vet 1994: 4).

\subsection{Prédicats transitionnels et non-transitionnels}

Vet (1994) distingue deux catégories de prédicats : les prédicats «transitionnels » et les prédicats " non-transitionnels ». Dans la classe des prédicats transitionnels, il oppose les " accomplissements " (ou procès complexes) aux « achèvements » (procès qui se réduisent à la transition même qui est, elle, de nature atomique/ponctuelle/momentanée). Parmi les procès non-transitionnels, on trouve les "processus ", les " positions » et les «états ». Les processus et les positions sont de nature temporaire, les états de nature permanente.

Le tableau (2) contient des exemples (repris de Vet 1994) qui illustrent les différents types de prédicats et leur combinaisons possibles ou impossibles avec les compléments de durée introduits par pendant et en.

\begin{tabular}{|l|l|}
\hline Type de prédicat & Exemple \\
\hline Achèvement & Son arrivée a surpris Pierre *pendant/*en une demi-heure. \\
\hline Accomplissement & Jeanne a planté un arbre *pendant/en une demi-heure. \\
\hline Processus & Dorothée a couru pendant/*en deux heures aujourd'hui. \\
\hline Position & Dorothée a gardé son argent pendant/*en deux heures. \\
\hline État & Dorothée a été bête *pendant/*en deux heures. \\
\hline
\end{tabular}

Tableau (2) : Illustration des différentes combinaisons (im)possibles avec les compléments de durée introduits par pendant et en

Quand on analyse la capacité des prédicats à tolérer l'aspect perfectif (qui «se combine avec des procès bornés » (Vet 1994: 11)) et l'aspect imperfectif (qui implique qu'une partie seulement du procès est achevée, et non pas sa totalité), 
on peut déduire que les « achèvements » et les " accomplissements » sont téliques (c'est-à-dire que ces prédicats présentent des actions ou des événements comme menés à terme) ; ce sont des procès transitionnels qui se combinent aisément avec l'aspect «perfectif » et moins facilement avec l'aspect « imperfectif ». En revanche, les « processus » et les « positions » (qui ont en commun «le fait d'être conceptualisés comme se référant à un ensemble de procès identiques [...], des procès homogènes » (Vet 1994: 7)) sont plutôt non-téliques ; ce sont des procès non-transitionnels, qui se combinent difficilement avec l'aspect " perfectif » et aisément avec l'aspect « imperfectif ». Enfin, les « états » (qui se distinguent des processus et des positions par le fait qu’ils « désignent une propriété ou une relation conçue comme étant de nature permanente » (Vet 1994: 7)) ne sont ni téliques ni non-téliques, car ces prédicats ne décrivent pas une action ou un événement qui pourrait éventuellement aboutir à son terme.

\subsection{La télicité de à travers (de) au XVI siècle}

Stosic a remarqué que le caractère non-relationnel de à travers dans son usage en français moderne « ressort également du fait que cette préposition permet de décrire plutôt des procès non téliques » (2005: 210). Une phrase comme :

(7) Les soldats se promènent/courent/circulent/errent/défilent/marchent à travers la ville.

montrerait que à travers se combine surtout avec des verbes médians qui expriment un déplacement à l'intérieur d'un même site.

En principe, l'expression à travers (de) construit (avec le verbe) une relation locative de polarité médiane : autrement dit, elle exprime «la phase du parcours » et localise une cible « pendant le déplacement » (Stosic 2005: 208). De plus, la relation « est vraie tout au long de la phase [médiane] de déplacement » (Borillo 1998: 42). Il en résulte que à travers est, en français moderne, une préposition dynamique qui ne peut contribuer qu'à exprimer une relation de même nature. Stosic semble donc fondé à décrire l'usage actuel comme étant plutôt non-télique.

Cependant, qu'en était-il au XVI ${ }^{\mathrm{e}}$ siècle ? Tout comme en français moderne, la majorité des configurations présentent des prédicats non-téliques (des « processus » dans la classification de Vet) ; mais il y a aussi des exceptions. Tout d'abord, l'exemple (8) montre qu'on rencontre des mouvements fictifs (non perceptuels) qui sont présentés comme étant dynamiques par la langue mais qui, dans la réalité, se révèlent statiques dès lors que le prédicat exprime un « état ». Comme le montre l'exemple suivant, on a affaire à une opposition frontale évidente au déplacement fictif de la cible ${ }^{7}$ :

\footnotetext{
7 Comme souvent dans le cas d'un mouvement fictif, la cible est une entité fictive ici, qui n’est pas mentionnée dans le contexte linguistique ; c’est la probe en anglais (voir Talmy 2000)
} 
(8) Donques nous entendons par expérience, que de mile lieues de la pomme de la terre, l'on doit compter dix autres mile lieues au neufiesme ciel : et de cent lieues du globe de la terre, mile lieues d'un pole à l'autre, et à travers de la terre y ha neuf mil quatre cens cinquante lieues : lesquelz pôles sont joignants au ciel empirée, et par eux tournent les deux sphères l'une au contraire de l'autre.

(Alphonse Jean, dit Fonteneau, Voyages avantureux du Capitaine Jan Alfonce, Sainctongeois, 1544)

Un autre usage de à travers (de) au $\mathrm{XVI}^{\mathrm{e}}$ siècle serait aujourd'hui paraphrasé au moyen d'en travers (de). Il s'agit de la configuration où, dans le domaine marin, se voit décrite une position géographique orthogonale. Si l'on suit Stosic, à travers (de) aurait ici "gardé son sens d'origine (travers est issu du latin transversus - « oblique, transversal ») » (Stosic 2002a: 152), en ce sens que la localisation géographique de la cible indiquée par à travers (de) se trouve sur une ligne orthogonale à l'axe principal de l'objet pris en référence (le site). Dans ce genre d’usage, le prédicat exprime de nouveau un état :

(9) Apres y ha le rocher, qui est à travers de Blanchbergues, l'isle de Lestraple ${ }^{8}$, le banch de Caraque, puis les banchs de l'Escluse, où entre la rivière qui passe à Dan et à Bruges. ${ }^{9}$

(Alphonse Jean, dit Fonteneau, Voyages avantureux du Capitaine Jan Alfonce, Sainctongeois, 1544)

Outre ces exemples où le prédicat décrit un état, on rencontre encore, au $\mathrm{XVI}^{\mathrm{e}}$ siècle, des configurations où le site sert de lien entre différentes entités, et où le prédicat revêt donc une nature télique. Quand il est vrai que le mouvement passe toujours par l'intérieur du site (voir Stosic 2002b: 117-118), celui-ci est destiné au passage ou, en tout cas, l'admet facilement. Comme pour les sites que sélectionne par, il peut s'agir d'objets de type « conduit » qui sont destinés à faciliter le passage, et qui se prêtent donc à un procès de transition. Par conséquent, les prédicats qui sélectionnent ce genre de site sont aussi transitionnels. Nous avons rencontré au moins deux configurations où une telle valeur télique est exprimée :

a) Site fonctionnellement destiné au passage :

8 « Terstreep (aussi appelé Ter Streep ou Testerep) est le nom d’une ancienne île qui était située sur la côte belge, avant que le bras de mer entre l'île et la côte ne s'ensable [...]. À partir du XII siècle, on a drainé la zone au moyen d'écluses » (wikipedia).

9 Dans Dassié (1677: 324) on retrouve une description similaire de l'actuelle côte belge ; remarquons que à travers de est de nouveau utilisé pour localiser le rocher : « Entre Calais et Zelande la coste gist Est Ouest, fort dangereuse des bancs, et entre autres les bancs de Gravelines puis les bancs de Nieuwport, le banc de Caraque, puis les bancs de l'Escluse; à travers de Blanchebergues il y a un rocher, [...] ». 
(10) Or pour vous faire entendre le nom de cognaté, cest oiseau est de telle nature, que mangeant quelque chose qui lui blesse l'estomach, subit le rejette par le cul, ne luy faisant que passer à travers les boyaux, ce que fit cette première grue ;

(Philippe d'Alcripe, La Nouvelle fabrique des excellents traicts de verité,

1580-1596)

Le site peut alors prendre les formes suivantes :

- objet de type « conduit » (tuyau, circuit, conduit, etc.) (cf. exemple 10) ;

- voie de communication conceptualisée comme «bordée » (tunnel, rue, etc.) ;

- entité-site de la catégorie des « ouvertures » (trou, fenêtre ouverte, etc.).

b) Site non fonctionnellement destiné au passage (mais qui l'admet facilement) :

(11) J'aymerois bien à voir en Xenophon une telle louange d'Agesilaus : estant prié par un prince voisin avec lequel il avoit autresfois esté en guerre de le laisser passer en ces terres, il l'octroya, luy donnant passage à travers le Peloponnesse;

(Michel de Montaigne, Essais, 1592)

Le site peut alors être :

- un lieu de type « agglomération », de type «pays » ou de type «paysage » (une ville, un jardin...), au singulier ou au pluriel (cf. exemple 11) ;

- une collection, un ensemble d'objets (c'est-à-dire que la cible parcourt des entités qui « sont envisagé[e]s comme formant un tout » (Stosic 2009: 20)) ;

- une entité mixte (se comportant parfois comme un lieu, parfois comme un objet ; je n'ai pas rencontré d'exemples de cet emploi au XVI ${ }^{\mathrm{e}}$ siècle).

Remarquons, enfin, que le sens du verbe est un facteur important pour l'identification d'un procès. Il n'est par conséquent pas étonnant d'arriver à une lecture télique dans des occurrences où à travers (de) se combine avec un verbe télique comme passer. Stosic va jusqu'à affirmer qu'une lecture télique de à travers (de) n'est jamais due à la sémantique de la préposition même, mais plutôt « à l'interaction entre les propriétés lexicales du verbe et le caractère perfectif du passé composé ou d'un autre temps grammatical du même type » (2002b: 184).

\section{CONCLUSION}

Dans cette contribution nous avons proposé une analyse sémantique de l'usage qui était fait, au $\mathrm{XVI}^{\mathrm{e}}$ siècle, de la locution française à travers (de). En nous basant sur les descriptions de Stosic, qui traite de l'usage spatial contemporain de à travers et qui a introduit la notion de "guidage ", nous avons donné un aperçu condensé des emplois, illustrés par des exemples provenant de FRANTEXT. 
Ensuite, en nous fondant sur la typologie de Vet (1994), nous avons analysé quelques occurrences dans lesquelles la nature télique ou non-télique du procès en cause peut prêter à discussion. Pour l'usage de à travers $(d e)$ au $\mathrm{XVI}^{\mathrm{e}}$ siècle, notre analyse semble confirmer la thèse de Stosic, selon qui à travers décrit plutôt des procès non-téliques en français moderne. Cependant, on rencontre aussi des exceptions, notamment des combinaisons avec des prédicats qui expriment des procès téliques, ou bien des états.

Notre travail montre comment des analyses de la langue actuelle peuvent servir de point de départ pour l'étude d'un état de langue plus ancien. Dans le même temps, cette démarche permet à mieux comprendre la structure sémantique actuelle de l'expression visée en dégageant des convergences et des divergences entre la situation d'aujourd'hui et celle du XVI siècle.

Afin de corroborer les résultats, une analyse portant sur les périodes qui séparent le $\mathrm{XVI}^{\mathrm{e}}$ siècle de l'époque actuelle, et si possible sur la période précédente, s'impose. On pourra ainsi mieux capter l'évolution sémantique, et plus spécifiquement celle de la nature télique ou non-télique, de notre expression.

\section{REFERENCES}

Borillo A., 1998, L'espace et son expression en français, Gap/Paris, Editions Ophrys.

Dassié, F., 1677, L'architecture Navale, avec le routier des Indes Orientales et Occidentales, Paris, Jean de la Caille.

FRANTEXT, UMR 7118 ATILF/Nancy2, <http://www.frantext.fr>.

Herskovits A., 1986, Language and spatial cognition: An interdisciplinary study of the prepositions in English, Cambridge, Cambridge University Press.

Hoelbeek T., 2014. The spatial expressions containing French 'travers' and Italian 'traverso'. A functional semantic description from a diachronic perspective, thèse de Doctorat, Bruxelles, Vrije Universiteit Brussel/Université Libre de Bruxelles.

Sarda L., 1999, Contribution à l'étude de la sémantique de l'espace et du temps : analyse des verbes de déplacement transitifs directs du français, thèse de Doctorat, Toulouse, Université de Toulouse-Le Mirail.

Somers A., 1988, 'En travers', une analyse sémantique, mémoire de Licence, Bruxelles, Vrije Universiteit Brussel.

Stosic D., 2002a, Les prépositions spatiales 'par' et 'à travers' - convergences et divergences, in L. Kupferman (ed.), La préposition française dans tous ses états - 4, Actes du Colloque PREP AN 2000, Scolia 15, p. 143-156.

Stosic D., 2002b, 'Par' et 'à travers' dans l'expression des relations spatiales : comparaison entre le français et le serbo-croate, thèse de Doctorat, Toulouse, Université de Toulouse-Le Mirail.

Stosic D., 2005, 'Prendre par le sentier à travers le bois' ou comment 'à travers' (se) fraie un chemin, M. Tenchea \& A. Tihu (eds), Prépositions et conjonctions de subordination. Actes du Colloque franco-roumain de linguistique (Timisoara, 29-31 mai 2001), Syntaxe et sémantique, Timisoara, Editura Excelsior, p. 207-218. 
Stosic D., 2007, The Prepositions 'par' and 'à travers' and the Categorization of Spatial Entities in French, in M. Aurnague, M. Hickmann \& L. Vieu (eds), The Categorization of Spatial Entities in Language and Cognition, Human Cognitive Processing 20, Amsterdam/Philadelphia, p. 71-92.

Stosic D., 2009, Comparaison du sens spatial des prépositions 'à travers' en français et 'kroz' en serbe, Langages 173, p. 15-33.

Stosic D., 2012. Le pouvoir cadratif des compléments introduits par 'à travers’: des cadres de discours pas comme les autres? Travaux de linguistique 64(1), p. 55-78.

Talmy L., 2000, Toward a cognitive semantics. Volume 1: Concept structuring systems, Cambridge (Mass.)/London, The MIT Press.

Vandeloise C., 1986, L'espace en français, Paris, Éditions du Seuil.

Vendler Z., 1957, Verbs and Times, The Philosophical Review 66, p. 143-160.

Vendler Z., 1967, Linguistics in Philosophy, Ithaca (NY), Cornell University Press.

Vet C., 1994, Petite grammaire de l'aktionsart et de l'aspect, Cahiers de grammaire 19, p. 1-18. 